\title{
Aspect-Level Deep Collaborative Filtering via Heterogeneous Information Networks
}

\author{
Xiaotian Han ${ }^{1}$, Chuan Shi ${ }^{1 *}$, Senzhang Wang ${ }^{2}$, Philip S. Yu ${ }^{3,4}$, Li Song ${ }^{1}$ \\ ${ }^{1}$ Beijing University of Posts and Telecommunications \\ ${ }^{2}$ Nanjing University of Aeronautics and Astronautics \\ ${ }^{3}$ University of Illinois at Chicago \\ ${ }^{4}$ Institute for Data Science, Tsinghua University \\ \{hanxiaotian.h,song200626\}@gmail.com,shichuan@bupt.edu.cn, \\ szwang@nuaa.edu.cn,psyu@cs.uic.edu
}

\begin{abstract}
Latent factor models have been widely used for recommendation. Most existing latent factor models mainly utilize the rating information between users and items, although some recently extended models add some auxiliary information to learn a unified latent factor between users and items. The unified latent factor only represents the latent features of users and items from the aspect of purchase history. However, the latent features of users and items may stem from different aspects, e.g., the brandaspect and category-aspect of items. In this paper, we propose a Neural network based Aspect-level Collaborative Filtering model (NeuACF) to exploit different aspect latent factors. Through modelling rich objects and relations in recommender system as a heterogeneous information network, NeuACF first extracts different aspect-level similarity matrices of users and items through different meta-paths and then feeds an elaborately designed deep neural network with these matrices to learn aspect-level latent factors. Finally, the aspect-level latent factors are effectively fused with an attention mechanism for the top-N recommendation. Extensive experiments on three real datasets show that NeuACF significantly outperforms both existing latent factor models and recent neural network models.
\end{abstract}

\section{Introduction}

Currently the overloaded online information overwhelms users. In order to tackle the information overload problem, Recommender Systems (RS) are widely employed to guide users in a personalized way of discovering products or services they might be interested in from a large number of possible alternatives. Due to its importance in practice, recommender systems have been attracting remarkable attention in both industry and academic research community.

\footnotetext{
${ }^{*}$ Corresponding author.
}

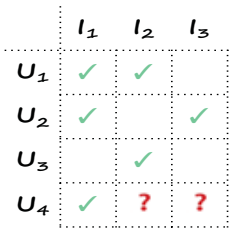

(a) User-Item purchase relation

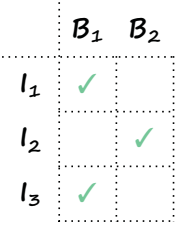

(b) Item-Brand relation

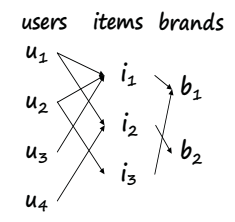

(c) heterogeneous network
Figure 1: A toy example of aspect-level interactions between users and items.

Collaborative Filtering (CF) [Hu et al., 2008] is the most popular method for recommendation, whose basic assumption is that people who share similar purchase in the past tend to have similar choices in the future. In order to exploit users' similar purchase preference, latent factor models (e.g., matrix factorization) [Koren et al., 2009; Koren, 2008] have been proposed, which usually factorize the user-item interaction matrix (e.g., rating matrix) into two low-rank user-specific and item-specific factors, and then use the low-rank factors to make predictions. Since latent factor models may suffer from data sparsity, many extended latent factor models integrate auxiliary information under the matrix factorization framework, such as social recommendation [Ma et al., 2008] and heterogeneous network based recommendation [Shi et al., 2016]. Recently, with the surge of deep learning, deep neural networks are also employed to deeply capture the latent features of users and items for recommendation. NeuMF [He et al., 2017] replaces the inner product operations in matrix factorization with a multi-layer feed-forward neural network to capture the nonlinear relationship between users and items. DMF [Xue et al., 2017] uses the rating matrix directly as the input and maps user and items into common low-dimensional space via a deep neural network.

Although these latent factor models achieve good performance, we find that they usually only capture the information of purchase history. Existing models usually focus on exploiting latent factors of users and items through their interaction information (especially rating information), which 
only reflects user preferences and item characteristics from one aspect, i.e., purchase history. However, the latent factors of users and items usually stem from different aspects in real applications. These different aspect-level features can more comprehensively reflect user preferences and item characteristics. Thus the latent factor models should exploit latent features of users and items from different aspects. Figure 1 shows a toy example of our idea. If we only exploit the interaction matrix (illustrating purchase history) in Figure 1a, we may infer that user $U_{4}$ will purchase item $I_{2}$ and $I_{3}$. However, when considering the item brand information shown in Figure $1 \mathrm{~b}$, we may find item $I_{3}$ is a better recommendation to $U_{4}$ because items $I_{1}$ and $I_{3}$ belong to the same brand $B_{1}$.

Although it is promising to comprehensively utilize multiple aspect-level latent features of users and items, it still faces the following two challenges. (1) How to extract different aspect-level features. We need to effectively organize the different types of objects and interactions in RS. The extracted aspect-level features should reflect different aspects of users preferences and embody rich semantics. (2) How to learn and fuse latent factors from different aspects. Even if we can extract different aspect-level features, it is still not easy to learn their latent factors and effectively fuse them. Although matrix factorization is an option, it only learns the "shallow" factors. Deep neural network is a promising method, while we still need to design proper network structure and fusing mechanism for our problem setting.

In this paper, to address the challenges above, we propose a novel Neural network based Aspect-level Collaborative Filtering model (NeuACF). NeuACF can effectively model and fuse different aspect-level latent factors which represent the user preferences and item characteristics from different aspects. Particularly, the objects and interactions of different types in RS are organized as a Heterogeneous Information Network (HIN) [Shi et al., 2017b]. Meta-paths [Sun et al., 2011], relation sequences connecting objects, are employed to extract features of users and items in different aspects. As an example shown in Figure 1c, we can extract the latent factors of users from the aspect of purchase history with the $U$ ser-Item-User path, which is usually analyzed by existing latent factor models. Furthermore, we design a delicate deep neural network to learn different aspect-level latent factors for users and items and utilize an attention mechanism to effectively fuse them for the top- $\mathrm{N}$ recommendation. Note that, different from focusing on the rating information with the auxiliary information in those hybrid recommendation models [Wang et al., 2015], NeuACF treats different aspect-level latent factors extracted with meta-paths equally, and automatically determines the importance of these aspects. NeuACF is also different from those HIN based methods [Yu et al., 2014; Shi et al., 2017a] in its deep model and fusing mechanism. Extensive experiments illustrate the effectiveness of NeuACF, as well as the traits of aspect-level latent factors.

\section{Preliminaries}

\subsection{Latent Factor Model}

The latent factor model has been widely studied in recommender system. Its basic idea is to map users and items

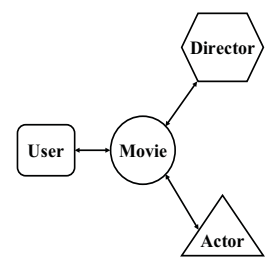

(a) MovieLens

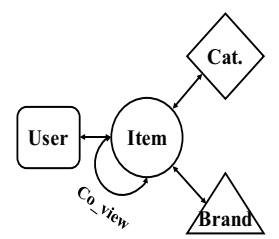

(b) Amazon
Figure 2: Network schema of HINs for the experimental datasets.

to latent factors and use these factors for recommendation. The representative works are Matrix Factorization (MF) [Koren et al., 2009], PMF [Mnih and Salakhutdinov, 2008] and SVD++ [Koren, 2008]. Taking the MF for example, the objective function of $\mathrm{MF}$ in Equation 1 aims to minimize the regularized squared loss on the observed ratings:

$$
\underset{\boldsymbol{u}, \boldsymbol{v}}{\arg \min } \sum_{i} \sum_{j}\left(\boldsymbol{R}_{i, j}-\boldsymbol{u}_{i}^{T} \boldsymbol{v}_{j}\right)^{2}+\lambda\left(\sum_{i}\left\|\boldsymbol{u}_{i}\right\|_{2}^{2}+\sum_{j}\left\|\boldsymbol{v}_{j}\right\|_{2}^{2}\right)
$$

where $\boldsymbol{u}_{i}$ and $\boldsymbol{v}_{j}$ denote the latent factors of user $U_{i}$ and item $I_{j} . \quad \lambda$ controls the strength of regularization, which is usually a $L-2$ norm aiming to prevent overfitting.

Based on this basic MF framework, many extended latent factor models have been proposed through adding some auxiliary information, such as social recommendation [Ma et al., 2008] and heterogeneous network based recommendation [Shi et al., 2015]. The limitation of existing latent factor models is that the latent factors are mainly extracted from one aspect, i.e., the rating matrix. However, some other more fine-grained aspect-level user-item interaction information is largely ignored, although such information is also useful.

\subsection{Heterogeneous Information Network}

The recently emerging HIN [Shi et al., 2017b] is a good way to model complex objects and relations in RS. Particularly, $\mathrm{HIN}$ is a special kind of information network, which either contains multiple types of objects or multiple types of links. The network schema of a HIN specifies the type constraints on the sets of objects and relations among the objects. Two examples used in experiments are shown in Figure 2. In addition, meta-path [Sun et al., 2011], a relation sequence connecting objects, can effectively extract features of objects and embody rich semantics. For example, in Figure $2 b$, the meta-path $U$ ser-Item-User $(U I U)$ extracts the features of users in the purchase history aspect, which means users having the same purchase records. A HIN-based recommendation model was first proposed by [Yu et al., 2014]. After that, several HIN based recommendations [Shi et al., 2017a; Zhao et al., 2017] have been proposed to utilize rich heterogeneous information in RS, while they usually focus on rating prediction with the "shallow" model. 


\begin{tabular}{l|c|c|c}
\hline \multirow{2}{*}{ Datasets } & \multirow{2}{*}{ Aspect } & \multicolumn{2}{|c}{ Meta-Paths } \\
\cline { 3 - 4 } & & User & Movie/Item \\
\hline \multirow{3}{*}{ MovieLens } & History & $U M U$ & $M U M$ \\
\cline { 2 - 4 } & Director & $U M D M U$ & $M D M$ \\
\cline { 2 - 4 } & Actor & $U M A M U$ & $M A M$ \\
\hline \multirow{3}{*}{ Amazon } & History & $U I U$ & $I U I$ \\
\cline { 2 - 4 } & Brand & $U I B I U$ & $I B I$ \\
\cline { 2 - 4 } & Category & $U I C I U$ & $I C I$ \\
\cline { 2 - 4 } & Co_view & $U I V I U$ & $I V I$ \\
\hline
\end{tabular}

Table 1: Meta-paths used in experiments and the corresponding aspects.

\section{The NeuACF Model}

\subsection{Model Framework}

As we have discussed, existing latent factor models generally focus on learning one aspect of latent factors (e.g., rating interaction), but ignore other aspects. In this work, we propose a Neural network based Aspect-level Collaborative Filtering (NeuACF) model for the top-N recommendation. The basic idea of NeuACF is to extract different aspect-level latent features for users and items, and then learn and fuse these latent factors with deep neural network. The model contains three major steps. First, we construct a HIN based on the rich user-item interaction information in $\mathrm{RS}$, and compute the aspect-level similarity matrices under different meta-paths of HIN which reflects different aspect-level features of users and items. Next, a deep neural network is designed to learn the aspect-level latent factors separately by taking these similarity matrices as inputs. Finally, the aspect-level latent factors are combined with an attention component to obtain the overall latent factors for users and items. Next we will elaborate the three steps in the following subsections.

\subsection{Aspect-level Similarity Matrix Extraction}

We employ HIN to organize objects and relations in RS, due to its power of information fusion and semantics representation [Shi et al., 2015]. Furthermore, we utilize metapath to extract different-aspect features of users and items. Taking Figure $2 \mathrm{~b}$ as an example, we can use $U I U$ and $I U I$ paths to extract features of users and items on the purchase history aspect, which is extensively exploited by existing latent factor models. In addition, we can also extract features from other aspects. Table 1 shows more aspect examples in our experimental datasets.

Given a specific meta-path, there are several alternatives to extract the aspect-level features: commuting matrix or similarity matrix. In this paper, we employ the similarity matrix based on the following reasons. (1) Similarity measure can alleviate noisy information; (2) Similarity values within the $[0,1]$ range are more suitable for learning latent factors. We employ the PathSim [Sun et al., 2011] to calculate aspectlevel similarity matrices under different meta-paths in our experiments. For example, we compute the similarity matrices of user-user and item-item based on the meta-paths $U I B I U$ and $I B I$ for the brand-aspect features respectively.

\subsection{Learning Aspect-level Latent Factors}

With the computed user-user and item-item similarity matrices of different aspects, we next learn their latent factors. Different from previous HIN based recommendation models,

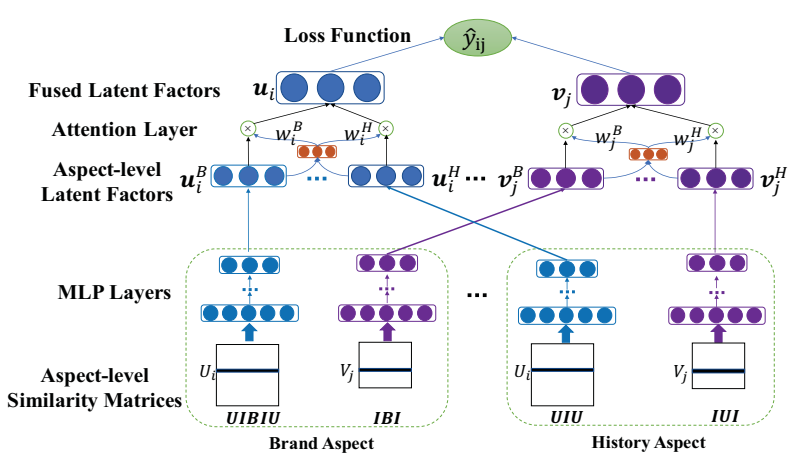

Figure 3: Deep neural network in the NeuACF model.

we design a deep neural network to learn their corresponding aspect-level latent factors separately, and the model architecture is shown in Figure 3. Concretely, for each user in each aspect, we extract the user's similarity vector from the aspectspecific similarity matrix. Then we take the similarity matrix as the input of the Multi-Layer Perceptron (MLP) and MLP learns the aspect-level latent factor as the output. The item latent factors of each aspect can be learned in a similar way.

Take the similarity matrix $S^{B} \in \mathbb{R}^{N \times N}$ of users under the meta-path $U I B I U$ as an example. User $U_{i}$ is represented as an $N$-dimensional vector of $S_{i *}^{B}$, which means $U_{i}$,s similarity to all the other users. Here the $N$ means the total number of users in the datasets. The MLP projects the initial similarity vector $S_{i *}^{B}$ of user $U_{i}$ to a low-dimensional aspect-level latent factor. In each layer of MLP, the input vector is mapped into another vector in a new space. Formally, given the initial input vector $S_{i *}^{B}$, and the hidden layer $\boldsymbol{H}_{l}$ where the $l$ is the $l$ th layer, the final aspect-level latent factor $\boldsymbol{u}_{i}^{B}$ can be learned through the following multi-layer mapping functions.

$$
\begin{aligned}
& \boldsymbol{H}_{\mathbf{0}}=\boldsymbol{S}_{i *}^{B} \\
& \boldsymbol{H}_{\mathbf{1}}=f\left(\boldsymbol{W}_{1}^{T} * \boldsymbol{H}_{0}+\boldsymbol{b}_{1}\right) \\
& \cdots \\
& \boldsymbol{H}_{\boldsymbol{l}}=f\left(\boldsymbol{W}_{l}^{T} * \boldsymbol{H}_{l-1}+\boldsymbol{b}_{l}\right) \\
& \cdots \\
& \boldsymbol{u}_{i}^{B}=f\left(\boldsymbol{W}_{n}^{T} * \boldsymbol{H}_{n-1}+\boldsymbol{b}_{n}\right)
\end{aligned}
$$

where $\boldsymbol{W}_{i}$, and $\boldsymbol{b}_{i}$ are the weight matrix and bias for the $i$ th layer, respectively, and we use the $\operatorname{Re} L U$, i.e., $f(x)=$ $\max (0, x)$ as the activation function in the hidden layers. From the learning framework in Figure 3, one can see that for each aspect-level similarity matrix of both users and items there is a corresponding MLP learning component described above to learn the aspect-level latent factors.

\subsection{Attention based Aspect-level Latent Factors Fusing}

After the aspect-level latent factors are learned separately for users and items, next we need to integrate them together to obtain aggregated latent factors. A straightforward way is to concatenate all the aspect-level latent factors to form a higher-dimensional vector. Another intuitive way is to average all the latent factors. The issue is 
that both methods do not distinguish their different importance because not all the aspects contribute to the recommendation equally (we will show that in the experiment part). Therefore, we choose the attention mechanism to fuse these aspect-level latent factors. Attention mechanism has been shown effective in various machine learning tasks such as image captioning and machine translation [You et al., 2016; Bahdanau et al., 2014]. The advantage of attention mechanism is that it can learn to assign attentive weights (normalized by sum to 1) for all the aspect-level latent factors: higher (lower) weights indicate that the corresponding features are informative (less informative) for recommendation. Specifically, given the user's brand-aspect latent factor $\boldsymbol{u}_{i}^{B}$, we use a two-layer network to compute the attention scores $s_{i}^{B}$ by the following Equation 3.

$$
\boldsymbol{s}_{i}^{B}=\boldsymbol{W}_{2}^{T} f\left(\boldsymbol{W}_{1}^{T} * \boldsymbol{u}_{i}^{B}+\boldsymbol{b}_{\mathbf{1}}\right)+\boldsymbol{b}_{\mathbf{2}}
$$

where $\boldsymbol{W}$ is the weight matrix and $\boldsymbol{b}$ is the bias.

The final attention weights for the aspect-level latent factors are obtained by normalizing the above attentive scores with the Softmax function given in Equation 4, which can be interpreted as the contributions of different aspects $a$ to the aggregated latent factor of user $U_{i}$.

$$
\boldsymbol{w}_{i}^{a}=\frac{\exp \left(\boldsymbol{s}_{i}^{a}\right)}{\sum_{n \in \mathbb{A}} \exp \left(\boldsymbol{s}_{i}^{n}\right)}
$$

Here, $\mathbb{A}$ is the set of all the aspects.

After obtaining all the attention weights $\boldsymbol{w}_{i}^{a}$ of all the aspect-level latent factors for user $U_{i}$, the aggregated latent factor $\boldsymbol{u}_{i}$ can be calculated by the Equation 5 .

$$
\boldsymbol{u}_{i}=\sum_{a \in \mathbb{A}} \boldsymbol{w}_{i}^{a} \cdot \boldsymbol{u}_{i}^{a}
$$

\subsection{Model Optimization}

We model the top-N recommendation as a classification problem which predicts the probability of interaction between users and items in the future. In order to ensure that the output value is a probability, we need to constrain the output $\hat{y}_{i j}$ in the range of $[0,1]$, where we use a Logistic function as the activation function for the output layer. The probability of the interaction between the user $U_{i}$ and item $I_{j}$ is calculated according to Equation 6.

$$
\hat{y}_{i j}=\operatorname{sigmod}\left(\boldsymbol{u}_{i} * \boldsymbol{v}_{j}\right)=\frac{1}{1+e^{-\boldsymbol{u}_{i} * \boldsymbol{v}_{j}}}
$$

where $\boldsymbol{u}_{i}$ and $\boldsymbol{v}_{j}$ are the aggregated latent factors of user $U_{i}$ and item $I_{j}$ respectively.

Over all the training set, according to the above settings, the likelihood function is

$$
p\left(\mathcal{Y}, \mathcal{Y}^{-} \mid \Theta\right)=\prod_{i, j \in \mathcal{Y}} \hat{y}_{i j} \prod_{i, k \in \mathcal{Y}^{-}}\left(1-\hat{y}_{i k}\right)
$$

where the $\mathcal{Y}$ and the $\mathcal{Y}^{-}$are the positive and negative instances set, respectively. The $\Theta$ is the parameters set.

Then we take the negative logarithm of the likelihood function Equation 7 to get the point-wise loss function in Equation 8 .

$$
\text { Loss }=\sum_{i, j \in \mathcal{Y} \cup \mathcal{Y}^{-}}\left(y_{i j} \log \hat{y}_{i j}+\left(1-y_{i j}\right) \log \left(1-\hat{y}_{i j}\right)\right)
$$

\begin{tabular}{c|c|c|c|c}
\hline Dataset & \#users & \#items & \#ratings & \#density \\
\hline ML100K & 943 & 1682 & 100,000 & $6.304 \%$ \\
\hline ML1M & 6040 & 3706 & $1,000,209$ & $4.468 \%$ \\
\hline Amazon & 3532 & 3105 & 57,104 & $0.521 \%$ \\
\hline
\end{tabular}

Table 2: The statistics of the datasets.

where $y_{i j}$ is the ground truth of the instance and $\hat{y}_{i j}$ is predicted score. This is the objective function to minimize in our model, and we can optimize it by stochastic gradient descent or its variants.

\section{Experiments}

\subsection{Experimental Settings}

\section{Datasets}

We evaluate the proposed model over the publicly available MovieLens dataset [Harper and Konstan, 2016] and Amazon dataset [He and McAuley, 2016; McAuley et al., 2015]. The network schema is shown in Figure 2, and the statistics of the datasets are summarized in Table 2.

- MovieLens-100K (ML100k)/MovieLens-1M (ML1M) 1. MovieLens datasets have been widely used for movie recommendation. We used the version ML100K and ML1M. For each movie, we crawl the director, actor of the movie from IMDb.

- Amazon ${ }^{2}$ : This dataset contains users' rating data in Amazon. In our experiment, we select the items of Electronics categories for evaluation.

\section{Evaluation Metric}

We adopt the leave-one-out method [He et al., 2017; Xue et al., 2017] for evaluation. The latest rated item of each user is held out for testing, and the remaining data for training. Following previous works [He et al., 2017; Xue et al., 2017], we randomly select 99 items that are not rated by the user as negative samples and rank the 100 sampled items for the user. For a fair comparison with the baseline methods, we use the same negative sample set for each (user, item) pair in the test set for all the methods. We evaluate the model performance through the Hit Ratio (HR) and the Normalized Discounted Cumulative Gain (NDCG) defined in Equation 9.

$$
H R=\frac{\# \text { hits }}{\# \text { users }}, N D C G=\frac{1}{\# \text { users }} \sum_{i=1}^{\# \text { users }} \frac{1}{\log _{2}\left(p_{i}+1\right)}
$$

where \#hits is the number of users whose test item appears in the recommended list and $p_{i}$ is the position of the test item in the list for the $i$-th hit. In our experiments, we truncated the ranked list at $K \in[5,10,15,20]$ for both metrics.

\section{Baselines}

Besides two basic methods (i.e., ItemPop and ItemKNN), the baselines include two MF methods (MF and eALS), one pairwise ranking method (BPR), and two neural network based

\footnotetext{
${ }^{1}$ https://grouplens.org/datasets/movielens/

${ }^{2}$ http://jmcauley.ucsd.edu/data/amazon/
} 
methods(DMF and NeuMF). In addition, we also adopt a recent HIN based method (FMG) as baseline, since most HIN based methods are designed for rating prediction.

- ItemPop. Items are simply ranked by their interaction popularity.

- ItemKNN. It is a standard item-based collaborative filtering method.

- MF [Koren et al., 2009]. Matrix factorization is a representative latent factor model.

- eALS [He et al., 2016]. It is a state-of-the-art MF method for recommendation with the square loss.

- BPR [Rendle et al., 2009]. The Bayesian Personalized Ranking approach optimizes the MF model with a pairwise ranking loss.

- DMF [Xue et al., 2017]. DMF uses the interaction matrix as the input and maps users and items into a common low-dimensional space using a deep neural network.

- NeuMF [He et al., 2017]. It combines the linearity of MF and non-linearity of DNNs for modelling user-item latent structures.

- FMG [Zhao et al., 2017]. It proposes "MF+FM" framework for the HIN-based rating prediction. We modify its optimization object as point-wise ranking loss for the top-N recommendation.

\section{Implementation}

We implement the proposed NeuACF based on Tensorflow [Abadi et al., 2016]. We use the same hyper-parameters for all the datasets. For the neural network, we use a two-layer MLP with each hidden layer having 600 hidden units. The dimension of latent factors is 64 . We randomly initialized the model parameters with a xavier initializer [Glorot and Bengio, 2010], and used the Adam [Kingma and Ba, 2014] as the optimizer. We set the batch size to 1024 and set the learning rate to 0.0005 . When training our model, 10 negative instances are sampled for each positive instance. Table 1 illustrates the extracted aspects and corresponding meta-paths. The optimal parameters for baselines are set according to literatures. All the experiments are conducted on a machine with two GPUs (NVIDIA GTX-1080 *2) and two CPUs (Intel Xeon E5-2690 * 2).

\subsection{Experiment Results \\ Performance Analysis}

Table 3 shows the experiment results of different methods. One can observe that, NeuACF almost achieves all the best performance over all the datasets and criteria. As the newest model with neural network, NeuMF also performs well on most conditions, while NeuACF consistently outperforms NeuMF in almost all the cases with only one exception. We think the reasons lie in that multiple aspects of latent factors learned by NeuACF provide more overall features of users and items. Although FMG also utilizes the same features with NeuACF, the better performance of NeuACF implies that the deep neural network in NeuACF may have the better ability to learn latent factors of users and items than the "shadow" model in FMG.

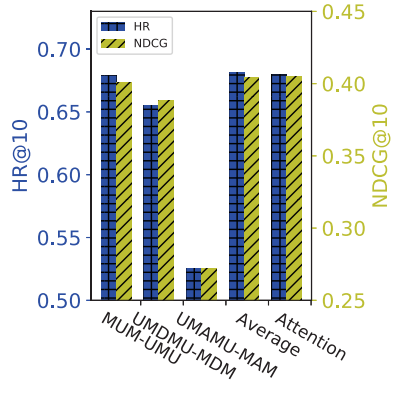

(a) ML100K

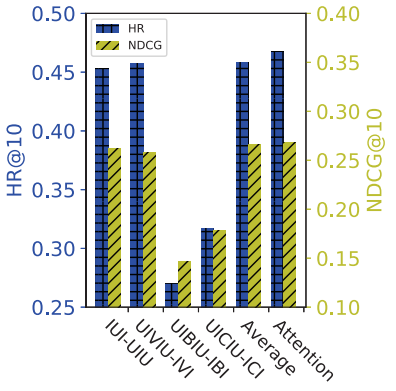

(b) Amazon
Figure 4: The impact of different aspect-level latent factors.

\section{Impact of Different Aspect-level Latent Factors}

To analyze the impact of different aspect-level latent factors on the algorithm performance, we run NeuACF with individual aspect-level latent factor through setting metapath. In Figure 4, for example, $U I B I U-I B I$ means that we only learn the brand-aspect latent factor for users and items. In addition, we also run NeuACF with the "Average" and "Attention" fusion mechanism, where "Average" means averaging all the aspect-level latent factors and "Attention" means fusing latent factors with the proposed attention mechanism. From the results shown in Figure 4, one can observe that the purchase-history aspect factors (e.g., $U I U-I U I$ and $U M U-M U M)$ usually get the best performance in all the individual aspects because this aspect usually contains the most important information which indicates the purchase history of users and items. One can also see that "Average" and "Attention" always perform better than individual meta-path, demonstrating fusing all the aspect-level latent factors can improve the performance. In addition, the better performance of "Attention" than "Average" also shows the benefit of the attention mechanism in NeuACF.

\section{Visualization of Different Aspect-level Latent Factors}
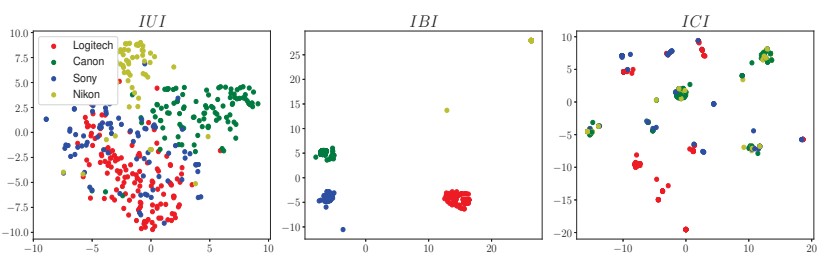

(a) t-SNE embedding with Brand labels

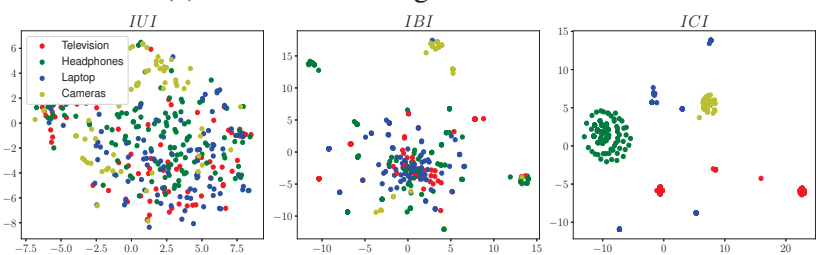

(b) t-SNE embedding with Category labels

Figure 5: t-SNE embedding with different labels of the learned latent factors of items for Amazon. 
Proceedings of the Twenty-Seventh International Joint Conference on Artificial Intelligence (IJCAI-18)

\begin{tabular}{|c|c|c|c|c|c|c|c|c|c|c|}
\hline Datasets & Metrics & ItemPop & ItemKNN & MF & eALS & BPR & DMF & NeuMF & FMG & NeuACF \\
\hline \multirow{8}{*}{ ML100K } & "HR@5 & 0.2831 & 0.4072 & 0.4634 & 0.4698 & 0.4984 & 0.3483 & 0.4942 & 0.4602 & 0.5097 \\
\hline & NDCG@5 & 0.1892 & 0.2667 & 0.3021 & 0.3201 & 0.3315 & 0.2287 & 0.3357 & 0.3014 & 0.3505 \\
\hline & HR@10 & 0.3998 & 0.5891 & 0.6437 & 0.6638 & 0.6914 & 0.4994 & 0.6766 & 0.6373 & 0.6846 \\
\hline & NDCG@10 & 0.2264 & 0.3283 & 0.3605 & 0.3819 & 0.3933 & 0.2769 & 0.3945 & 0.3588 & 0.4068 \\
\hline & HR@15 & 0.5366 & 0.7094 & 0.7338 & 0.7529 & 0.7741 & 0.5873 & 0.7635 & 0.7338 & 0.7813 \\
\hline & NDCG@15 & 0.2624 & 0.3576 & 0.3843 & 0.4056 & 0.4149 & 0.3002 & 0.4175 & 0.3844 & 0.4318 \\
\hline & HR@20 & 0.6225 & 0.7656 & 0.8144 & 0.8155 & 0.8388 & 0.6519 & 0.8324 & 0.8006 & 0.8464 \\
\hline & NDCG@20 & 0.2826 & 0.3708 & 0.4034 & 0.4204 & 0.4302 & 0.3151 & 0.4338 & 0.4002 & 0.4469 \\
\hline \multirow{8}{*}{ ML1M } & HR@5 & 0.3088 & 0.4437 & 0.5111 & 0.5353 & 0.5414 & 0.4892 & 0.5485 & 0.4732 & 0.5630 \\
\hline & NDCG@5 & 0.2033 & 0.3012 & 0.3463 & 0.3670 & 0.3756 & 0.3314 & 0.3865 & 0.3183 & 0.3944 \\
\hline & HR@10 & 0.4553 & 0.6171 & 0.6896 & 0.7055 & 0.7161 & 0.6652 & 0.7177 & 0.6528 & 0.7202 \\
\hline & NDCG@10 & 0.2505 & 0.3572 & 0.4040 & 0.4220 & 0.4321 & 0.3877 & 0.4415 & 0.3767 & 0.4453 \\
\hline & HR@15 & 0.5568 & 0.7118 & 0.7783 & 0.7914 & 0.7988 & 0.7649 & 0.7982 & 0.7536 & 0.8018 \\
\hline & NDCG@15 & 0.2773 & 0.3822 & 0.4275 & 0.4448 & 0.4541 & 0.4143 & 0.4628 & 0.4034 & 0.4667 \\
\hline & HR@20 & 0.6409 & 0.7773 & 0.8425 & 0.8409 & 0.8545 & 0.8305 & 0.8586 & 0.8169 & 0.8540 \\
\hline & NDCG@20 & 0.2971 & 0.3977 & 0.4427 & 0.4565 & 0.4673 & 0.4296 & 0.4771 & 0.4184 & 0.4789 \\
\hline \multirow{8}{*}{ Amazon } & HR@5 & 0.2412 & 0.1897 & 0.3027 & 0.3063 & 0.3296 & 0.2693 & 0.3117 & 0.3216 & 0.3268 \\
\hline & NDCG@5 & 0.1642 & 0.1279 & 0.2068 & 0.2049 & 0.2254 & 0.1848 & 0.2141 & 0.2168 & 0.2232 \\
\hline & HR@10 & 0.3576 & 0.3126 & 0.4278 & 0.4287 & 0.4657 & 0.3715 & 0.4309 & 0.4539 & 0.4686 \\
\hline & NDCG@10 & 0.2016 & 0.1672 & 0.2471 & 0.2441 & 0.2693 & 0.2179 & 0.2524 & 0.2595 & 0.2683 \\
\hline & HR@15 & 0.4408 & 0.3901 & 0.5054 & 0.5065 & 0.5467 & 0.4328 & 0.5258 & 0.5430 & 0.5591 \\
\hline & NDCG@15 & 0.2236 & 0.1877 & 0.2676 & 0.2647 & 0.2908 & 0.2332 & 0.2774 & 0.2831 & 0.2924 \\
\hline & HR@20 & 0.4997 & 0.4431 & 0.5680 & 0.5702 & 0.6141 & 0.4850 & 0.5897 & 0.6076 & 0.6257 \\
\hline & NDCG@20 & 0.2375 & 0.2002 & 0.2824 & 0.2797 & 0.3067 & 0.2458 & 0.2925 & 0.2983 & 0.3080 \\
\hline
\end{tabular}

Table 3: HR@K and NDCG@K comparisons of different methods.

In our model, we aim to learn the aspect-level latent factors from different meta-paths. For example, we expect that the brand-aspect latent factor $\boldsymbol{v}_{j}^{B}$ for item $I_{j}$ can be learned from the meta-path $I B I$, and the category-aspect latent factor $\boldsymbol{v}_{j}^{C}$ from the meta-path $I C I$. To intuitively show whether NeuACF performs well on this task, we visualize the learned aspect-level latent factors on the Amazon dataset. We apply t-SNE [Maaten and Hinton, 2008] to embed the highdimensional aspect-level latent factors into a 2-dimensional space, and then visualize each item as a point.

Figure 5a shows the embedding result for four famous electronics Brand: Logitech, Canon, Sony, and Nikon. One can observe that the brand-aspect latent factors can clearly separate the four brands, while the history-aspect and categoryaspect latent factors are mixed with each other. It demonstrates the meta-path $I B I$ can learn a good brand-aspect latent factors. Similarly, in Figure 5b, only the category-aspect latent factors learned from the meta-path $I C I$ clearly separate the items of different categories including Television, Headphones, Laptop and Cameras. The results demonstrate that the aspect-level latent factors of items learned by NeuACF can indeed capture the aspect characteristics of items.

\section{Effect of the Latent Factor Dimension}

In the latent factor models, the dimension of the latent factors may have a vital impact on the performance of recommendation. Thus we study the effect of dimension of the latent factor learned from the last MLP layer in our proposed model. We conduct the experiments on a 2-layer model, and set the dimensions of the latent factors increasing from 8 to 256. The results on the ML100k and Amazon datasets are shown in Figure 6. One can see that on both cases the perfor-

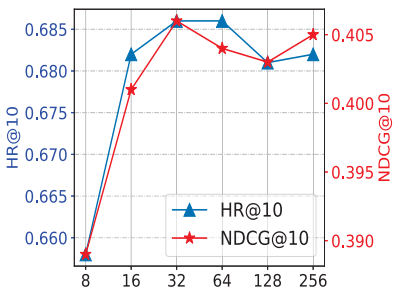

(a) ML100k

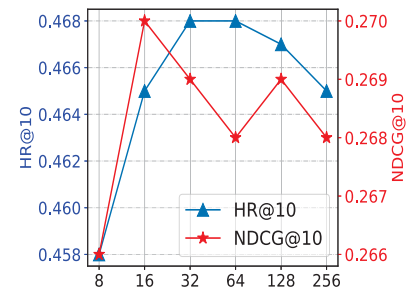

(b) Amazon
Figure 6: Performance with different dimension of latent factors.

mance first increases with the increase of the dimension, and the best performance is achieved at around 16-32. Then the performance drops if the dimension further increases.

\section{Conclusion and Future Work}

In this work, we explore aspect-level information for collaborative filtering and propose a Neural network based Aspectlevel Collaborative Filtering model (NeuACF). Based on different-aspect features extracted from heterogeneous network with meta-paths, the NeuACF learns aspect-level latent factors with a well-designed deep neural network and then fuses them with an attention mechanism for the top-N recommendation. Extensive evaluations demonstrate the superior performance of NeuACF.

As future work, we would like to utilize better attention mechanisms to fuse aspect-level latent factors. In addition, we can explore the strategy of automatic selection of metapaths in different datasets. 


\section{Acknowledgements}

This work is supported in part by the National Natural Science Foundation of China (No. 61772082, 61602237, 61672313, 61320106006), the National Key Research and Development Program of China (2017YFB0803304), and the Beijing Municipal Natural Science Foundation (4182043). This work is also supported in part by NSF through grants IIS-1526499, IIS-1763325, and CNS-1626432.

\section{References}

[Abadi et al., 2016] Martín Abadi, Ashish Agarwal, Paul Barham, Eugene Brevdo, Zhifeng Chen, Craig Citro, Greg S Corrado, Andy Davis, Jeffrey Dean, Matthieu Devin, et al. Tensorflow: Large-scale machine learning on heterogeneous distributed systems. arXiv preprint arXiv:1603.04467, 2016.

[Bahdanau et al., 2014] Dzmitry Bahdanau, Kyunghyun Cho, and Yoshua Bengio. Neural machine translation by jointly learning to align and translate. arXiv preprint arXiv:1409.0473, 2014.

[Glorot and Bengio, 2010] Xavier Glorot and Yoshua Bengio. Understanding the difficulty of training deep feedforward neural networks. In AISTATS, pages 249-256, 2010.

[Harper and Konstan, 2016] F Maxwell Harper and Joseph A Konstan. The movielens datasets: History and context. ACM Transactions on Interactive Intelligent Systems (TiiS), 5(4):19, 2016.

[He and McAuley, 2016] Ruining He and Julian McAuley. Ups and downs: Modeling the visual evolution of fashion trends with one-class collaborative filtering. In $W W W$, pages 507-517, 2016.

[He et al., 2016] Xiangnan He, Hanwang Zhang, Min-Yen Kan, and Tat-Seng Chua. Fast matrix factorization for online recommendation with implicit feedback. In SIGIR, pages 549-558. ACM, 2016.

[He et al., 2017] Xiangnan He, Lizi Liao, Hanwang Zhang, Liqiang Nie, Xia Hu, and Tat-Seng Chua. Neural collaborative filtering. In $W W W$, pages 173-182, 2017.

[Hu et al., 2008] Yifan Hu, Yehuda Koren, and Chris Volinsky. Collaborative filtering for implicit feedback datasets. In ICDM, pages 263-272. IEEE, 2008.

[Kingma and Ba, 2014] Diederik Kingma and Jimmy Ba. Adam: A method for stochastic optimization. arXiv preprint arXiv:1412.6980, 2014.

[Koren et al., 2009] Yehuda Koren, Robert Bell, and Chris Volinsky. Matrix factorization techniques for recommender systems. Computer, 42(8), 2009.

[Koren, 2008] Yehuda Koren. Factorization meets the neighborhood: a multifaceted collaborative filtering model. In SIGKDD, pages 426-434. ACM, 2008.

[Ma et al., 2008] Hao Ma, Haixuan Yang, Michael R Lyu, and Irwin King. Sorec: social recommendation using probabilistic matrix factorization. In CIKM, pages 931940. ACM, 2008.
[Maaten and Hinton, 2008] Laurens van der Maaten and Geoffrey Hinton. Visualizing data using t-sne. Journal of machine learning research, 9(Nov):2579-2605, 2008.

[McAuley et al., 2015] Julian McAuley, Christopher Targett, Qinfeng Shi, and Anton Van Den Hengel. Image-based recommendations on styles and substitutes. In SIGIR, pages 43-52. ACM, 2015.

[Mnih and Salakhutdinov, 2008] Andriy Mnih and Ruslan R Salakhutdinov. Probabilistic matrix factorization. In NIPS, pages 1257-1264, 2008.

[Rendle et al., 2009] Steffen Rendle, Christoph Freudenthaler, Zeno Gantner, and Lars Schmidt-Thieme. Bpr: Bayesian personalized ranking from implicit feedback. In $U A I$, pages 452-461, 2009.

[Shi et al., 2015] Chuan Shi, Zhiqiang Zhang, Ping Luo, Philip S Yu, Yading Yue, and Bin Wu. Semantic path based personalized recommendation on weighted heterogeneous information networks. In CIKM, pages 453-462. ACM, 2015.

[Shi et al., 2016] Chuan Shi, Jian Liu, Fuzhen Zhuang, S Yu Philip, and Bin Wu. Integrating heterogeneous information via flexible regularization framework for recommendation. Knowledge and Information Systems, 49(3):835859, 2016.

[Shi et al., 2017a] Chuan Shi, Binbin Hu, Wayne Xin Zhao, and Philip $S$ Yu. Heterogeneous information network embedding for recommendation. arXiv preprint arXiv:1711.10730, 2017.

[Shi et al., 2017b] Chuan Shi, Yitong Li, Jiawei Zhang, Yizhou Sun, and S Yu Philip. A survey of heterogeneous information network analysis. TKDE, 29(1):17-37, 2017.

[Sun et al., 2011] Yizhou Sun, Jiawei Han, Xifeng Yan, Philip S Yu, and Tianyi Wu. Pathsim: Meta path-based top-k similarity search in heterogeneous information networks. VLDB, 4(11):992-1003, 2011.

[Wang et al., 2015] Hao Wang, Naiyan Wang, and Dit-Yan Yeung. Collaborative deep learning for recommender systems. In SIGKDD, pages 1235-1244. ACM, 2015.

[Xue et al., 2017] Hong-Jian Xue, Xin-Yu Dai, Jianbing Zhang, Shujian Huang, and Jiajun Chen. Deep matrix factorization models for recommender systems. IJCAI, 2017.

[You et al., 2016] Quanzeng You, Hailin Jin, Zhaowen Wang, Chen Fang, and Jiebo Luo. Image captioning with semantic attention. In CVPR, pages 4651-4659, 2016.

[Yu et al., 2014] Xiao Yu, Xiang Ren, Yizhou Sun, Quanquan Gu, Bradley Sturt, Urvashi Khandelwal, Brandon Norick, and Jiawei Han. Personalized entity recommendation: A heterogeneous information network approach. In WSDM, pages 283-292. ACM, 2014.

[Zhao et al., 2017] Huan Zhao, Quanming Yao, Jianda Li, Yangqiu Song, and Dik Lun Lee. Meta-graph based recommendation fusion over heterogeneous information networks. In SIGKDD, pages 635-644. ACM, 2017. 